\title{
HIGH-THROUHGPUT FILM-DENSITOMETRY:
}

\section{AN EFFICIENT APPROACH TO GENERATE LARGE DATA SETS}

Dieter Typke ${ }^{1}$, Robert A. Nordmeyer ${ }^{2}$, Arthur Jones ${ }^{2}$, Juyoung Lee ${ }^{1}$, Agustin Avila-Sakar ${ }^{3}$, Kenneth H. Downing ${ }^{1}$, and Robert M. Glaeser ${ }^{1,3}$

${ }^{1}$ Life Sciences Division, Donner Laboratory, Lawrence Berkeley National Laboratory, University of California, Berkeley, CA 94720

${ }^{2}$ Engineering Division, Lawrence Berkeley National Laboratory, University of California, Berkeley, CA 94720

${ }^{3}$ Department of Molecular and Cell Biology, University of California, Berkeley, CA 94720

Corresponding Author:

Robert M. Glaeser

Lawrence Berkeley National Laboratory

University of California, Berkeley

Berkeley, CA 94720

Phone: 510-642-2905

Fax: 510-486-6488 


\section{ABSTRACT}

A film-handling machine (robot) has been built which can, in conjunction with a commercially available film densitometer, exchange and digitize over 300 electron micrographs per day. Implementation of robotic film handling effectively eliminates the delay and tedium associated with digitizing images when data are initially recorded on photographic film. The modulation transfer function (MTF) of the commercially available densitometer is significantly worse than that of a high-end, scientific microdensitometer. Nevertheless, its signal-to-noise ratio $(\mathrm{S} / \mathrm{N})$ is quite excellent, allowing substantial restoration of the output to "near-to-perfect" performance. Due to the large area of the standard electron microscope film that can be digitized by the commercial densitometer (up to 10,000x13,680 pixels with an appropriately coded holder), automated film digitization offers a fast and inexpensive alternative to high-end CCD cameras as a means of acquiring large amounts of image data in electron microscopy.

Key Words: electron microscopy; film densitometer; film-handling robot; Nikon Super Coolscan 8000 ED; modulation transfer function; MTF; Wiener filter; signal-to-noise ratio; geometric distortions 


\section{INTRODUCTION}

Reconstruction of the three-dimensional structure of a large macromolecular complex from its projection-images (Frank, 1996; Frank, 2002; Subramaniam and Milne, 2004) requires the use of digitized images of a large number of particles. As the technology of single-particle reconstruction continues to improve, there is growing interest in scaling up the number of particle-images that are used for the reconstruction. The very first 3-D reconstruction that might be done in any new project will already use a few thousand particles, and successive stages of a project are likely to use many tens of thousands (Agrawal et al., 2004) or even more than 100,000 particles. Ultimately, a very high-resolution reconstruction might require as many as one million images of single particles in the initial data set (Henderson, 1995; Glaeser, 1999), even though the number of particles used for the reconstruction may then be reduced by selection of the best members in the set (Sander et al., 2003; Yonekura et al., 2003; Borgnia et al., 2004; Subramaniam and Milne, 2004).

If electron micrographs are recorded with a CCD camera (Faruqi and Subramaniam, 2000; Zhang et al., 2003) or other form of direct electronic readout, the data are digitized in "real time”, i.e., as the images are read out of the camera. When the images are recorded on photographic film, however, one must use a film densitometer to digitize the data. Since as many as thousands of particle-images might be obtained by digitizing only a few tens of films, manual operation of a film densitometer does not represent a significant bottleneck at an early stage of a project. When the desired number of particles is subsequently increased by a factor of ten or even a hundred, however, the work of digitizing individual films does create a significant bottleneck that limits the rate of progress of the work.

Photographic film nevertheless continues to be a highly attractive medium for recording data when large numbers of particle-images need to be collected. As will be explained in detail 
below, a single photographic film can be digitized as an array of over 10,000x13,000 pixels, if the appropriate holder is used. When thought of as an "area detector", then, one film can cover more than 30 times the area (and thus include more than 30 times as many particles) as can an image that is recorded with a $2 \mathrm{Kx} 2 \mathrm{~K}$ CCD camera. The difference is smaller for a $4 \mathrm{Kx} 4 \mathrm{~K}$ CCD camera, of course, but only if the ratio of pixel size to the point spread function in the latter remains as large as it is for film.

Because it is of such great value to use the large detector-area that is available on film, and at the same time it is becoming necessary to digitize large numbers of films as part of a single project, it is worthwhile to automate the process of digitizing a large number of films. It is realistic to think that over 200 films/day could be digitized with less than an hour of human effort, a task that might extend for over a week or more of dedicated human effort without automation.

We describe here one way in which the digitization of images recorded on standard 3.25x4 inch EM films has been automated. We have built a film-handling robot that can be described as consisting of two mechanical modules. One module carries individual films to and from the film holder (a modified version of one that is provided with the commercial film densitometer), and the second module emulates the human motion required to insert the film holder into the densitometer and then remove it again after the scan has been completed. The densitometer for which we have implemented this conceptual design is the commercially distributed Nikon Super Coolscan 8000 ED film densitometer. This densitometer uses a 10,000pixel wide tri-linear CCD array as the detector, across which the film is scanned in a direction perpendicular to the detector.

As part of our effort to automate film densitometry, we have also characterized the densitometer performance itself, the goal being to correct systematic errors in the data to the 
degree that this is possible (for similar measurements on other film scanners see Mitsuoka et al., 1997 and Hesse et al., 2000). The two properties that were of greatest interest to us were (1) the modulation transfer function (MTF) of the densitometer and, more importantly, the spectral signal-to-noise curve for the densitometer and (2) the geometric distortions introduced by the densitometer. In short, the MTF of the densitometer was found to be anisotropic, falling to 0.5 at about 0.37 the Nyquist spatial frequency limit in the worst direction. The signal-to-noise ratio of the densitometer remains high up to much higher frequencies, however. As a result we can recommend restoration of the signal out to spatial frequencies as high as 0.7 of the Nyquist limit, even in the worst direction. Geometric distortions introduced by the densitometer were found to be small, in the range of a few pixels out of 10,000. Since these distortions are deterministic, they could in principle be corrected computationally, but for single-particle work there is no need to do so, and for imaging of ordered arrays the corrections will be done in any case as part of the "unbending" operations that are needed to compensate for distortions introduced by the microscope or present within the specimen itself.

\section{MATERIALS}

Densitometer chosen for automated digitization

The Nikon Super Coolscan 8000 ED is a commercial densitometer that is designed for scanning a variety of color as well as black-and-white films. It uses high-efficiency light emitting diodes (red, green and blue LEDs) as a light source, a specially designed Nikkor ED lens system to relay the image of the trans-illuminated film onto the detector, and a tri-linear CCD array with $3 \times 10,000$ pixels. The smallest pixel size referred to the film corresponds to 4,000 dots per inch, or $6.35 \mu \mathrm{m}$ per pixel. 
A software package called "NikonScan" is delivered with the densitometer. The software provides the user with a set of default values for parameters related to the scanning operation, as well as the option of changing these parameters to user-defined values. As we document below, the value of "Gamma" should be set to 1.0 (more precisely 1.03) for any measurement where it is expected that the values of the densitometer output are linear in the transmittance, $\mathrm{T}$, of the micrograph. However, usually one is interested in the optical density, $\mathrm{D}$, which is proportional to the logarithm of the transmittance (i.e., $\mathrm{D}=\lg (1 / \mathrm{T})$ ), because this quantity is, at least for values below $\mathrm{D}=1$, to a good accuracy proportional to the electron exposure of film (see e.g. Zeitler, 1992). To our knowledge there is no setting of the Nikon for which the output would be proportional to the optical density (OD). Thus the conversion into OD has to be carried out afterwards. The default value of 4000 for the "Resolution" takes full advantage of the resolution of the linear CCD detector, but this value naturally produces very large data files. A smaller value of the resolution may therefore be a better choice if the full resolution of the densitometer is not needed. The selection of "Grayscale" within the "Grayscale/Color" option is self-evident for electron micrographs, and in addition we have standardized our use around the choice of "Positive” within the "Positive/Negative" (contrast) option, because this provides linearity of the logarithm of the output with the optical density of film. We have also standardized our use of the Nikon densitometer by accepting the default scan speed rather than specifying the "Superfine" mode, since the latter requires approximately twice as long to complete a scan. However, the superfine mode will be more appropriate for micrographs that have very low contrast, because these scans do not contain the weak background pattern that is seen perpendicular to the y-axis of images when the default speed is used, and which shows up as weak reflections at about 1/18th the Nyquist frequency on the meridian of the power spectrum. It is further recommended that 14 bit data depth be chosen in the "Scan Bit Depth" option. The value of "Analog Gain" 
may be set to a slightly negative value, e.g. -0.04 , to ensure that the light parts of the gray value histogram are not compressed into one single value. The amount of "Strip Film Offset" can be specified by the user in order to determine where on the holder the scan will begin, which is particularly useful when modified holders are used. The information about the position of the scan area is obtained from thumbnail images and is set by adjusting the "Boundary Offset" and then clicking the "Reload Thumbnail” button.

A number of film holders for different types and formats of film are delivered together with the Nikon scanner. Film types include formats ranging from 35-mm film and $5 \times 5 \mathrm{~cm}$ slides to larger formats (up to $6 \mathrm{~cm}$ x $9 \mathrm{~cm}$ films). Holders for different formats are recognized by the scanner by means of a hole code that is located on the front end of the holder. This code defines the area(s) that can be scanned with that specific holder. The particular holder that we have modified for use in the robot scanner (FH 869 S) allows one to scan areas of up to 8,964x13,176 pixels, corresponding to a film area of $56.9 x 83.7 \mathrm{~mm}^{2}$. In presenting our experimental characterization of the Nikon densitometer, we designate the direction along the linear CCD as the $\mathrm{x}$-axis, and the $\mathrm{y}$-axis is then parallel to the direction of the linear scan.

\section{Test-films used to characterize the densitometer performance}

One particularly important test film that we used to characterize the densitometer, referred to as a "transmission sinusoidal test pattern," was purchased from JML Optical Industries (Rochester, NY). This particular test film (part \# STP16805/000) contains a set of subareas of calibrated optical densities along with a set of sub-areas with well-defined sine wave modulation of the transmittance. The frequencies of the sine gratings vary from $0.375 \mathrm{~mm}^{-1}$ to 80 $\mathrm{mm}^{-1}$, the highest frequency being slightly beyond the Nyquist limit $\left(78.7 \mathrm{~mm}^{-1}\right)$ at the highest (4,000 dpi) resolution of the scanner. 
An additional test film was a "home-made” step wedge that was prepared on Kodak SO 163 photographic film by illuminating successive areas of the film with a linearly increasing electron exposure. This "electron-exposure step wedge" was obtained by stepwise shifting the edge of the illuminated area, as defined by the selected area diffraction aperture, across the image plane, using the magnetic image shift. The film was exposed for $1 \mathrm{~s}$ at each position of the edge, resulting in a linear increase of the exposures on adjacent film areas.

Three experimental electron micrographs were used to further characterize the use of the densitometer. In the first, photographic film (Kodak SO 163 film) was simply illuminated with a uniform electron exposure (no specimen) of $\sim 0.5$ electrons $/ \mu \mathrm{m}^{2}$ (300 keV) and developed in full-strength D19 developer without antifog. The mean optical density of this film was about 0.5. In the second, an electron micrograph was recorded (at a magnification of 60,000x and $300 \mathrm{~nm}$ defocus using $400 \mathrm{keV}$ electrons) of a thin crystal of paraffin $\left(\mathrm{C}_{44} \mathrm{H}_{90}\right)$ supported on a thin carbon film. The electron exposure of the film was again $\sim 0.5$ electrons $/ \mu \mathrm{m}^{2}$. In the third, the specimen was a carbon film with evaporated gold islands, and the image was recorded on film at 400,000x magnification and $1 \mu \mathrm{m}$ underfocus using $200 \mathrm{keV}$ electrons.

\section{DESIGN OF THE FILM-HANDLING ROBOT}

The basic design for automated loading and removal of photographic films is organized around a mechanical unit that pushes the Nikon film holder from a resting position into the starting position (after which the film holder is moved in the normal way by the Nikon hardware and software.) The same unit also withdraws the film holder to a resting position after it is ejected by the densitometer, i.e., when a scan has been completed. A second mechanical unit loads films onto the film holder when it is in its resting position, and then removes them again after the scan has been completed. Integration of the software control (for these mechanical units) with the 
software control for the Nikon densitometer required having access to the Nikon Software Developer's Kit (SDK), generously provided by Nikon Europe.

In one cycle of film exchange, a single film is picked up from an input stack by a robotic hand that is equipped with suction fingers, and then the film is carried over to the empty holder. After this film has been released onto the glass "bottom plate" of the modified film holder (described below), a second glass plate is placed on top of the film by a separate robotic hand. At the end of the scanning cycle the top glass plate is lifted away, the film is picked up and placed into an output stack, and the whole system is again ready to repeat the cycle. Figure 1 shows a photograph of the entire system in order to illustrate the relative positions of each of the components mentioned above.

The Nikon film holder has been modified extensively for use in the automated operations just described. As is shown in Figure 2, the interior area of the commercially supplied holder (identification number FH 869 S) was cut away and replaced by a glass plate whose area is larger than that of a photographic film. Steel "shim stock" strips were then glued to the glass on either side of the intended position of the EM film. The thickness (i.e., height) of these strips is approximately $25 \mu \mathrm{m}$ greater than that of the film itself. The top glass plate then rests directly on the strips, and it is held to the strips by magnets mounted into the top glass plate. The result is that the film is not able to buckle or curl appreciably, while at the same time the gap between the top glass plate and the film is large enough to preclude the introduction of interference fringes due to reflection of light at closely juxtaposed surfaces. The fabrication and assembly of this modified holder was done with some care so as to ensure that all parts of the scanned negative would pass over the linear CCD without a change in focus. Uniformity of focus was, in turn, verified by measuring the MTF in different sub-areas over the entire scan. 
One entire cycle of film-transfer and scanning takes approximately 4.5 minutes. Of this time, 100 seconds are required for the robotic transfer of film and 160 seconds are required to scan 13,000 lines. More than 300 films can thus be scanned in 24 hours. Detailed shop drawings and a list of all commercially purchased components are available from www. [This URL will be added after the manuscript has been accepted for publication]. A movie showing the operation of the robot can also be viewed from that web page.

\section{CHARACTERIZATION OF THE DENSITOMETER PERFORMANCE}

In choosing the Nikon $8000 \mathrm{ED}$ densitometer as the instrument to automate, we were concerned about characterizing (a) the linearity of its response as a function of input film-density values, (b) the modulation transfer function (MTF) of the instrument, (c) the signal-to-noise ratio in the output, expressed as a function of spatial frequency, and (d) geometric distortions in the output scan. The approaches that we have used to characterize these properties of the densitometer are straightforward and easily replicated in any electron microscopy laboratory. In addition, the same approaches can just as easily be applied to any other type of film densitometer.

\section{a. Linearity of the logarithm of the densitometer output as a function of input film-density values}

The densitometer output consists of a file of numbers that represent the light intensity transmitted through the photographic film. The primary data are, of course, the intensity of light detected by the individual pixels of the CCD detector. These primary data are then automatically corrected to reflect the calibrated sensitivity of each pixel (and the incident light intensity of the light source at that pixel). The output data delivered by the scanner correspond to some function of the transmittance, $\mathrm{T}$, which depends upon the value of Gamma. For Gamma $=1.0$, the output 
was found to be proportional to $\mathrm{T}$ (more precisely to $\mathrm{T}^{1.03}$ ), while for the default value Gamma = 2.2, the output is proportional to $\mathrm{T}^{0.48}$. This function is further limited to a restricted range of values that is determined when the "Autoexposure" procedure of the scanner is executed. Finally the data are expanded in a proprietary way by the Nikon software into the range of 2-byte integer numbers $(-32768$ through +32767$)$. To make sure that the full transmittance range $0 \leq \mathrm{T} \leq 1$ is covered - which is required for a reliable conversion into optical densities - we recommend to run "Autoexposure" on an area that contains very light and very dark sub-areas. The transmittance range determined in this step is used for subsequent scans if "Autoexposure" is not checked in the pull-down menu "Preferences/Single Scans".

In the automatic scanning routines of the robot, neither "Autofocus" nor "Autoexposure" is executed. Instead we have chosen to run these as separate procedures before running the robot in the automatic mode.

Characterization of the linearity of the logarithm of the Nikon 8000 ED densitometer output as a function of the optical density was performed for a number of values of the Gamma factor, including the default setting of Gamma $=2.2$. The logarithm of the values recorded in the Nikon output (after shifting the data range to positive numbers) was compared to the optical density values stated by the manufacturer for the density steps in the JML test film. These measurements, shown in Figure 3A, demonstrate that the logarithm of the Nikon output is indeed strictly linear with the stated optical densities of the test film over a range of input densities from 0.0 to 1.4. The constant of proportionality depends upon the value of Gamma, of course, the slope being 0.48 for the default setting Gamma $=2.2$, and 1.03 (i.e., close to the expected value 1.0) for Gamma $=1.0$. In order to obtain a densitometer output that is linear in the transmitted light intensity (i.e., the film transmittance), one has, according to these measurements, to use the value Gamma $=1.03$. 
In an additional experiment we determined the dependence of the logarithm of the Nikon output on the electron exposure of photographic film for our recommended Gamma value of 1.0 using the "electron exposure step wedge" described above (see Figure 3B). Essentially perfect linearity was observed up to an optical density of about 0.7 , and no deviation from the linearity between exposure and OD could be recognized up to this OD level.

\section{b. The modulation transfer function (MTF) of the densitometer}

The JML test film was used as our "primary standard" for evaluating the MTF of the densitometer. This test film contains sub-areas in which the transmitted light intensity varies sinusoidally with defined amplitude, the value of which is stated in the manufacturer's data sheet that accompanies the test film. The densitometer output can be recorded as values that are proportional to the transmittance by using Gamma $=1.0$ - provided that the full transmittance range is covered during the "Autoexposure" procedure and after correction for the negative offset in the data. Alternatively, in order to compare the sinusoidal modulation of the Nikon output with the stated input modulation when the default value Gamma $=2.2$ is used, the output can be converted to transmittance using the fact that the slope in Figure $3 \mathrm{~A}$ is about 0.48 . In either case the Fourier transform of the digitized transmittance always produced power spectra for which the amplitudes of the harmonics were less than ca. $1.5 \%$ of the amplitude of the stated fundamental sinusoid. In addition, the amplitudes of the harmonics measured in the lowfrequency range were similar in magnitude to the values quoted in the data sheet for the test film.

The JML test film was digitized in two orthogonal orientations, one with the sine gratings parallel to the scan direction (the y-direction) and one with the gratings perpendicular to the scan direction. Use of the two orthogonal orientations thus allows one to separately measure the densitometer MTF in both the x-direction and in the y-direction. Figure 4 shows a series of 
discrete data points for the ratio of output to input amplitudes, corresponding to the individual, pure frequencies that are found within individual sub-areas of the test pattern. Although the amplitudes of the highest-frequency $\left(80 \mathrm{~mm}^{-1}\right)$ sine wave can, due to aliasing, only be measured at a frequency that is slightly lower than the Nyquist limit $\left(78.7 \mathrm{~mm}^{-1}\right)$, the measured value has nevertheless been plotted at its true frequency in Fig. 4. As the peaks are not confined to single pixels of the amplitude spectrum, they were integrated over a small ( $5 \times 5$ pixel) sub-area surrounding each peak (which has of course to be done in the power spectrum, since the phases of adjacent pixels are to be considered as independent random variables).

The photographic film that was exposed to a uniform (random) distribution of electrons served as a second test film. The input "signal" in this case might be modeled as a random distribution of delta functions, whose expected (average) amplitude spectrum is constant with spatial frequency. Because the film itself also has an imperfect frequency response, however, the Fourier spectrum of the input function that is presented to the densitometer is no longer constant, nor is it simple to characterize the input spectrum with a high degree of accuracy. The use of this type of test film can nevertheless provide an estimate of the combined MTF of film plus the densitometer, which is the product of the separate MTF of the film and that of the densitometer. It is ultimately the MTF of the entire detector system that is of interest, which, in this case, is the combined MTF of film plus densitometer and not just that of the densitometer. A comparison of the results obtained with the uniformly exposed film and those obtained with the pure sine grating input function will also provide interesting information on whether the point spread function of photographic film makes a significant contribution on top of that of the densitometer.

An accurate comparison of the MTF for the combined system against the MTF of just the densitometer alone requires that the noise introduced by the film-component of the detector system does not much affect the MTF that is determined in this way. The film noise is expected 
to consist of two components. The first of these is the fog of randomly developed silver grains that is present even in unexposed film. The physical size of these individual grains is presumably much smaller than the point spread function that characterizes the registration of incident electrons ((Zeitler, 1992)). The smaller point spread function associated with the fog density will, in turn, make a contribution to the combined MTF of the measurement that has correspondingly higher amplitudes at high frequencies than that generated by the exposure of film to electrons. Because the optical density of the fog is much lower than the OD $\sim 0.5$ that was produced by the incident electrons, however, we believe that its effect can be ignored in the measurements that we report here. The second component of the film noise arises from variations in the amount of film-blackening that is caused by individual electron events. The noise associated with variable pulse heights for individual electrons will be characterized by essentially the same point spread function of the film as is the case for the random distribution of the electron events themselves. As a result, film-noise that is due to a variation in the distribution of pulse heights will not misrepresent the component of the total system MTF that is due to the film itself, and thus this source of noise can also be ignored for our purposes here if the amplitude spectrum is appropriately scaled.

There is, nevertheless, an important flaw that still must be recognized in the use of a uniformly exposed test film. An unavoidable problem with such a test film is that its Fourier spectrum extends out to spatial frequencies that are much higher than the Nyquist limit of the densitometer. The high-frequency components of such a test film will therefore be aliased back at lower frequencies, thereby giving a too optimistic estimate of the combined MTF. Because the effect of aliasing cannot be corrected, we designate the MTF that is determined with the use of a uniformly illuminated film as the apparent MTF of the (combined) detector system. (For similar 
measurements on CCD cameras see (Meyer and Kirkland, 2000). These authors use the term “Noise Transfer Function” instead of “apparent MTF”.)

The apparent MTF of the combination of film plus scanner is shown in Figure 4A as two continuous curves (which are smoothed versions of the raw amplitude spectra of the digitized image) for the $\mathrm{x}$-direction and y-direction, respectively. As there is no absolute scaling of the amplitude spectrum, the curves were scaled within the spatial frequency range 10 to $20 \mathrm{~mm}^{-1}$ to coincide with the data points obtained earlier with the sine-grating input function. Once scaled in this way, the apparent MTF curves are actually quite close, except at the extreme low and high frequencies, to those obtained for the densitometer alone (obtained with pure sine-grating functions as the input).

The relatively high amplitudes that are observed at low spatial frequencies are unlikely to be due to structure (i.e., intensity variations) within the uniform electron beam. Instead, we suspect that they are due to the fact that the photographic film may have significant lowfrequency variations in sensitivity, due for example to non-uniform emulsion thickness or nonuniform development of the emulsion. These low-frequency variations in sensitivity then result in variations in film density even though the electron exposure was uniform. The deviation that appears at high frequency, on the other hand, is likely to have a major contribution from the aliasing effect discussed above.

It is obvious from Figure 4A that the MTF falls off much more rapidly in the x-direction (the direction of the line of CCD pixels) than it does in the y-direction (the direction in which the film is moved). The MTF in the y-direction falls to a value of 0.5 at a spatial frequency of approximately 36 cycles/mm, which is about $46 \%$ of the Nyquist frequency. Roughly speaking, therefore, the point spread function of the densitometer has a full width at half maximum of approximately $12 \mu \mathrm{m}$ in the y-direction. The MTF in the x-direction is significantly worse, 
however, falling to a value of 0.5 at a spatial frequency of approximately 29 cycles $/ \mathrm{mm}$, which is about $37 \%$ of the Nyquist frequency. As a result, the point spread function of the densitometer has a full width at half maximum of approximately $15 \mu \mathrm{m}$ in the x-direction. Although we have not investigated the reason for the observed anisotropy of the MTF, we can suggest a number of factors that may contribute. (1) The light sources (i.e., the set of red, green and blue LEDs) illuminate a narrow band of the film that is oriented parallel to the CCD array. This means that light emerging from an LED under one pixel may be scattered into one or more of the adjacent CCD pixels in the x-direction but not in the y-direction, causing cross-talk in the information recorded by pixels in the x-direction but not in the y-direction. (2) The diffraction-limited point spread function of the light-collection optics is fully sampled along the CCD direction, but only the central part of the Airy disk is sampled in the y-direction. (3) The electron-transfer efficiency of the CCD is less than $100 \%$, thereby causing some blurring of information (cross talk between pixels) along the CCD direction. (4) The signal contained in each CCD pixel must be read out through an amplifier and an analog-to-digital converter. The frequency response of these electronic components may reduce the MTF in the x-direction but not in the y-direction. In a preliminary evaluation of the Zeiss SCAI microdensitometer we have observed the same type of anisotropy of the MTF, with the resolution being better in the direction perpendicular to the linear CCD detector.

As a further characterization of the MTF of the Nikon densitometer, the same uniformly exposed negative was also digitized on a Perkin Elmer 1010M PDS microdensitometer, using a pixel size of $5 \mu \mathrm{m}$. The apparent MTF obtained with the PDS (up to the Nyquist frequency of the Nikon scanner) is shown in Figure 4B, along with modified versions of the apparent MTF curves for the Nikon densitometer described earlier. In this case the two experimental curves representing the apparent MTF for the Nikon (and that of the PDS densitometer, as well) were 
replaced by a smoothed fitting that goes to a value of 1 at zero frequency, on the assumption that the true MTF should be perfect at low frequency. The values of the apparent MTF curves for the Zeiss SCAI are intermediate between those of the Nikon 8000 ED and those of the Perkin Elmer PDS microdensitometer (data not shown). The apparent MTF of the PDS densitometer is approximately 0.6 at the Nyquist limit of the Nikon densitometer. This apparently favorable result is once again too optimistic an estimate of the MTF of the PDS densitometer, however, because (once again) the "shot noise" input function that was used is not band-limited. The important point about making this comparison is simply to show that the MTF of the Nikon is well below that of a precision microdensitometer, and to show that the MTF of the film does not contribute significantly to the apparent MTF of the Nikon densitometer. This latter point confirms the same conclusion that would be drawn from the close similarity of the apparent MTF to the values of the densitometer MTF that were measured with the use of a pure sine-grating test film.

\section{c. Signal-to-noise ratio of the densitometer output as a function of spatial frequency}

Once the MTF of a linear system has been characterized, one can - in principle - apply an inverse operation that will restore the output spectrum to give a better match between the input and the output of the system. There are two factors that make it impossible to achieve a completely perfect restoration of the input signal, however.

The first concern is the unwanted amplification of Fourier components that come from frequencies beyond the Nyquist limit, which are therefore aliased into the observed spectrum. In most applications the aliased Fourier components will actually be noise, since the spectrum of the desired object is itself likely to be band-limited but the noise will not be. Unfortunately, it is not possible to mathematically deconvolute the desired signal (i.e., the signal at frequencies below the Nyquist limit) from the Fourier components that are contributed by aliasing. Instead, 
the user must simply work at a high enough image magnification that the highest desired spatial frequency does not get too close to the Nyquist frequency, where aliasing might become significant.

A more important concern with the restoration procedure is the danger of amplification of noise that is introduced by the densitometer itself. The user must therefore decide at what value of the signal-to-noise ratio, $\mathrm{S} / \mathrm{N}$, the data can still be used, and then not apply a restoration operation beyond that point. An even better approach is to weight the restoration with a Wienertype filter that takes into account the frequency-dependent signal-to-noise ratio. We remark that amplification of noise (e.g. electron shot noise) that is already present in the input signal is not of concern, since the input noise is attenuated by the densitometer MTF in exactly the same way as is the desired input signal so that the signal-to-noise ratio is unchanged.

A characterization of the $\mathrm{S} / \mathrm{N}$ ratio (as a function of spatial frequency) in the densitometer output should therefore be obtained, in order to design an inverse operation that will restore the input as well as possible. The noise in the densitometer output is, by definition, whatever remains after one computes the difference between two otherwise identical scans of the same film. The S/N of the densitometer output can therefore be obtained by calculating the ratio of the moduli of the Fourier transforms of (1) one or the other of the two, "identical” scans and (2) the difference between them (divided by $\sqrt{2}$ ).

Figure 5 shows an example of the steps involved in performing such a restoration. Panel 5A shows the original (smoothed), one-dimensional amplitude-spectra for the digitized image of a uniformly exposed film, in two orthogonal directions (as in Figure 4B). In addition, Panel 5A also shows the corresponding amplitude spectra for the difference image, divided by $\sqrt{2}$, i.e. for the noise that is contributed by the densitometer itself. The noise was found to be very small indicating a high reproducibility of the Nikon scanner. Panel 5B then shows the smoothed, full 
2-D amplitude spectrum of the digitized image, while Panel 5C shows the corresponding, smoothed amplitude spectrum for the difference between two such scans. The grayscale representation of the noise spectrum that is shown in Panel 5C is greatly amplified relative to that of the amplitude itself, shown in Panel 5B, as may be seen by referring to the respective curves in Panel 5A.

Panel 5D shows smoothed, one-dimensional curves that represent the $\mathrm{S} / \mathrm{N}$ in two orthogonal directions, corresponding to the ratio of the noise and amplitude spectra shown in Panel 5A. The noise introduced by the Nikon densitometer is considerably smaller than the signal over the full frequency range, even in the $\mathrm{x}$-direction close to the Nyquist limit (i.e., the worst case). It is thus apparent that one can restore the digitized image data to a value that is close to the original value on the film over a wide range of the frequency spectrum. In order to perform the restoration as well as possible, we have created a Wiener filter that takes into account the $\mathrm{S} / \mathrm{N}$ of the densitometer. Curves showing the values of the Wiener filter, in two orthogonal directions, are also shown in Panel 5D, and the full two-dimensional version of this function is displayed in Panel 5E. The use of a Wiener filter produces a significant improvement in the apparent MTF, as is illustrated in Panel 5F. When using such a filter function, one has to keep in mind that the film noise was not taken into account in designing the filter. It thus remains present after restoration. An example of restoration of the MTF is shown in Figure 6. In this case the specimen consisted of monolayer crystals of paraffin $\left(\mathrm{C}_{44} \mathrm{H}_{90}\right)$ that had been grown on a thin carbon film. Panel A shows an area of the digitized image, with the 2-D amplitude spectrum inserted in the upper right-hand corner. Three pairs of diffraction spots corresponding to the 0.41 $\mathrm{nm}$ and $0.38 \mathrm{~nm}$ Bragg spacings in the paraffin crystal are visible in the spectrum. The spatial frequency of the Bragg reflections is approximately 55 percent of the Nyquist frequency. The fairly rapid falloff of the MTF of the densitometer is apparent in the continuous background of 
the amplitude spectrum. The MTF of the densitometer thus acts as a low-pass filter, the effect of which is to produce the coarse granularity of the background that can be seen in the digitized image. Panel B shows the same area after restoration of the signal, using the Wiener filter depicted in Panel 5E. The brightness of the Bragg reflections relative to that of the central spot is, of course, increased by restoration of the signal. The restoration of a more balanced amplitude spectrum also produces a much finer grain in the digitized image, as can be seen by comparing Panel A and Panel B. Indeed, achieving the best possible restoration of the original balance of amplitudes within the Fourier spectrum is the sole objective of applying an inverse filter, since the $\mathrm{S} / \mathrm{N}$ ratio cannot be improved by such an operation.

\section{d. Geometric distortions of the densitometer}

Geometric distortions can be introduced into the densitometer output in three distinct ways. The first possibility is that the step-size of the film movement in the y-direction might not be precisely the same as the pixel size in the $\mathrm{x}$-direction. To the extent that this is the case, the magnification in the digitized image will not be identical in the two orthogonal directions. The second possibility is that the relay optics might introduce off-axis geometric distortions, such as pincushion or barrel distortions. The most important concern in this case, when digitizing images of single particles, would be that the output magnification would not be constant over the digitized image. The third possibility is that the linear CCD detector may not be mounted at precisely 90 degrees relative to the scan direction. The result in this case would be to "shear" the image such that the square array of pixels displayed in the output image is actually derived from a rhombohedral array of sampling points in the input image.

All three of these potential, geometric distortions were characterized by analyzing two digitized versions of the same negative, which in this case contained an image of evaporated gold islands on a carbon film. In retrospect, we believe that an image of a clean carbon film 
would serve equally well for this purpose. The film was first scanned when mounted in the "portrait" orientation and then rotated by 90 degrees so that it was scanned in the "landscape" orientation. Since the angle of manual rotation of the negative is not expected to be precisely 90 degrees, the actual rotation angle was also determined as a parameter in the analysis. The "landscape" scan was first rotated back computationally by (exactly) 90 degrees so that specimen-features had the same orientation as they did in the portrait scan. The two images then share (almost) the same orientation and contain a square region of $8964 \times 8964$ pixels that corresponds to the same area of the original negative, as is indicated schematically in Figure 7A.

The common, square regions were first extracted from both of the (initially rectangular) scans, such that their centers coincide within one pixel. The analysis of geometric distortions then used a central row and a central column of 512x512 sub-areas, as is shown in Figure 7A. The relative position of each sub-area within the full, common (8964x8964) area was found by cross-correlation. The displacements found for each sub-area were then plotted as functions of distance from the center of the common area, as is shown in Figure 7B. The nomenclature used in Figure 7B should be understood as follows: a sub-area centered at point $(\mathrm{x}, 0)$ in the first scan will be found to be displaced by $\left(\Delta \mathrm{x}_{\text {peak }}(\mathrm{x}, 0), \Delta \mathrm{y}_{\text {peak }}(\mathrm{x}, 0)\right)$ in the second scan. Similarly, a subarea centered at the point $(0, y)$ in the first scan will be found to be displaced by $\left(\Delta \mathrm{x}_{\text {peak }}(0, \mathrm{y})\right.$, $\left.\Delta \mathrm{y}_{\text {peak }}(0, \mathrm{y})\right)$ in the second scan.

The curves for $\Delta \mathrm{y}_{\text {peak }}(\mathrm{x}, 0)$ and $\Delta \mathrm{x}_{\text {peak }}(\mathrm{y}, 0)$ are linear, however, their slopes turned out to be different. As the pixel size along the CCD direction is certainly constant in the central part of the image, the linearity of both curves can be taken as an indication that the pixel size is also constant along the y-axis, i.e. the $y$-movement is linear. This linearity was further confirmed by scanning the same film area at two different y-positions. The slopes of the curves $\Delta \mathrm{y}_{\text {peak }}(\mathrm{x}, 0)$ and $\Delta \mathrm{x}_{\text {peak }}(\mathrm{y}, 0)$ indicate the difference of orientations of the axes of both scans. While the 
relative displacement of $\Delta \mathrm{x}_{\text {peak }}(\mathrm{y}, 0)$ is close to zero over the full range, indicating that the y-axes coincide quite well, $\Delta \mathrm{y}_{\text {peak }}(\mathrm{x}, 0)$ increases to an amount of 18 pixels over the distance of 4096 pixels, indicating an angular difference of 4.4 mrad between the x-axes. Since the y-axes coincided almost perfectly, it can easily be shown that, as a coincidence in this case, half of the difference in this angle is due to a mis-orientation of the CCD with respect to the scanning direction, and the other half is due to the deviation from a 90-degree rotation of the film between the scans.

The displacements $\Delta \mathrm{x}_{\text {peak }}(\mathrm{x}, 0)$ and $\Delta \mathrm{y}_{\text {peak }}(0, \mathrm{y})$ provide information about differences in the pixel size along the $\mathrm{x}$ - and $\mathrm{y}$-directions. Only one of these curves is shown in Figure 7B, because the information contained in the other one is redundant. A cubic function can be fitted to the data that are shown in Figure $7 \mathrm{~B}$ for $\Delta \mathrm{x}_{\text {peak }}(\mathrm{x}, 0)$. The linear term of this polynomial function indicates the ratio of pixel sizes in the $\mathrm{x}$ - and $\mathrm{y}$-directions at the center of the scan area, while the third-order term indicates the distortion of the optical (lens) system. The relative pixel size difference at the center was found to be about 0.0005 pixels, the pixel being slightly compressed in the y-direction relative to the pixel size in the CCD direction. Due to a barrel distortion of the lens system, the relative pixel size difference increases to about 0.004 at a distance of 4,000 pixels from the center. In absolute terms the accumulated deviation of a detail at the edge of an image from its correct position is about 5 pixels.

\section{DISCUSSION}

The Nikon densitometer has a design that is well suited for automated exchange of photographic films

The key factors that we considered to be important in selecting a commercially available film densitometer for use with a film-handling robot were (1) the possibility to digitize as large 
an area of the standard electron microscope (EM) film as is possible, (2) availability of a digitized pixel size (or modulation transfer function) that is not wasteful of the relatively high resolution that is available in EM film, (3) high geometric precision and reproducibility, and small geometric distortion associated with digitization, (4) a system of holding and exchanging EM films that lends itself easily to emulation by a robot, and (5) an economical purchase cost.

The fact that the Nikon Super Coolscan 8000 ED film densitometer is at least an order of magnitude less expensive than many other instruments currently in use in EM laboratories, yet offers a relatively small pixel size and a very large pixel format, made this a logical instrument to consider more seriously as the basis for automated densitometry. Preliminary comparisons of the amplitude spectra of digitized versions of a few test films convinced us that this instrument would be a good choice in terms of the data that it produced. In addition, it was apparent that a simple mechanical system could be built to load and remove EM films for this instrument.

The apparent shortcomings of the Nikon densitometer can be compensated by application of systematic corrections

The relatively poor MTF of the Nikon densitometer relative to the Perkin Elmer PDS densitometer and even to the Zeiss SCAI scanner stands out as the principal shortcoming of the former. This shortcoming is further compounded by the fact that the MTF of the Nikon is not isotropic. In the end, however, it is the signal-to-noise $(\mathrm{S} / \mathrm{N})$ ratio as a function of spatial frequency, more than the MTF, that determines how well the information in a micrograph can be transferred from film to computer. Since the $\mathrm{S} / \mathrm{N}$ of the Nikon is greater than 4 out to about $80 \%$ of the Nyquist frequency, even in the worst direction (the x-direction), it is possible to restore the attenuated amplitude to a value much closer to what was originally present in the electron microscope image. A relatively good restoration can be achieved simply by dividing the 
amplitude spectrum of each image by the azimuthally dependent MTF obtained from uniformly exposed film. An even better restoration can be achieved by including the estimated $\mathrm{S} / \mathrm{N}$ ratio to compute a Wiener filter. Furthermore, in most applications it is wise, on other grounds, to oversample (at a given resolution) by using at least three rather than just two pixels per cycle. Assuming that such a degree of oversampling will be done, it is actually unnecessary to restore the signal beyond 2/3 of the Nyquist frequency. Thus, for most applications the restored signal that is obtained with the Nikon densitometer will be a "nearly perfect" representation of the desired information that was recorded on film.

Apart from the MTF, the performance of the Nikon densitometer is essentially perfect as regards its use for low-dose images of biological macromolecules. The logarithm of the Nikon output is linear with the electron exposure of the film over the range of densities that are normally encountered in such work. The geometric fidelity of the scan is also superb, and the very small geometric errors that do occur can be undone computationally, if desired.

Film is an efficient medium to use for recording large amounts of data

The primary advantage of recording data on film lies in the large number of pixels that are captured in each image. When using the FH 869 S film holder, for example, one can digitize EM films as an array of up to 8,964x13,176 pixels. Each such digitized image of an EM film thus contains over 7 times more data than a single $4 \mathrm{Kx} 4 \mathrm{~K}$ CCD image, and almost 30 times as much data as a single $2 \mathrm{Kx} 2 \mathrm{~K}$ CCD image. If all else were equal, therefore, the initial step of recording data could be completed far more rapidly on film than on currently available CCD cameras. 
In reality there are additional advantages and disadvantages involved in recording images by either method. As a result, a more accurate comparison between film and CCD cameras can become too complicated to discuss in any simple way. For example, automated data collection can (in principle) continue without interruption when using a CCD camera, whereas currently used film cameras require reloading after 50 exposures, and exchanging film boxes can slow the data collection substantially. Even this disadvantage of film recording could be eliminated by conversion to the use of a roll-film camera a step that would incidentally simplify the automation of film densitometry. As another example, CCD cameras have historically had rather large point spread functions relative to their pixel size, especially when used on electron microscopes with accelerating voltages greater than 100 kV (Daberkow, 1996; Downing and Hendrickson, 1999; Sherman et al., 1996). As a result, the usable number of pixels has been significantly less than the stated CCD array size of $2 \mathrm{Kx} 2 \mathrm{~K}$ or $4 \mathrm{Kx} 4 \mathrm{~K}$. Nevertheless, substantial improvements have been made in the performance of the most recent cameras when used at $120 \mathrm{kV}$ (Zhang et al., 2003), and additional improvements are currently being tested for cameras that are designed for voltages up to $300 \mathrm{kV}$. Needless to say, however, the cost of these high-end CCD cameras may limit their general accessibility.

Film recording, on the other hand, is a relatively inexpensive and universally available method of data collection. It is reasonable to assume that one person can record (and develop) at least $200 \mathrm{EM}$ films per session. In order to collect the same volume of data with a $4 \mathrm{Kx} 4 \mathrm{~K}$ CCD camera, which is the best case currently imagined, one would need to record at least 1500 frames of image data. Current systems for automated microscope operation can acquire this many CCD images in about one day of continuous operation (Zhu et al., 2001), and thus the throughput (in terms of human effort that is required) could be similar to that when film is used as the detector. Since the point spread function for CCD cameras is currently much worse at $300 \mathrm{kV}$ than at 100 
$\mathrm{kV}$, however, the number of CCD images that is required to obtain comparable amounts of data is far greater than the example above. These comparisons are presented less to say that one approach is markedly better than the other, but more to emphasize that film itself is a highly efficient medium for recording data.

The major drawback of using film has previously been the inefficiency of manual operation of a film densitometer. Manual digitization of as few as 20 films a day is generally regarded as being an onerous task, and it is not reasonable to consider scaling that effort up by a factor of 10. Thus, in order to take full advantage of the high efficiency that film offers for recording large data sets, it is sensible to turn to some scheme for fully automated, rapid digitization of EM films.

The robotic film-handler that is described in this paper, in combination with the Nikon 8000 ED densitometer, is able to digitize over 300 films per day with a human investment of time that is less than one hour. The adoption of automation for the film digitization process thus allows one to take full advantage of the high efficiency that film offers for recording large amounts of data. Indeed, automated digitization of EM film is likely to remain a sensible alternative to data-capture with $4 \mathrm{Kx} 4 \mathrm{~K}$ CCD cameras even when new technologies are able to deliver high performance cameras for $300 \mathrm{kV}$ electron microscopes.

\section{Acknowledgments}

We would like to thank Dr. Wah Chiu for scanning the JML test film and the uniformly exposed micrograph on the Zeiss SCAI scanner. We thank Nikon Europe for providing the Software Developer's Kit for the Nikon Super Coolscan 8000 ED. This work has been supported by NIH Grant GM 62989. 


\section{References}

Agrawal, R.K., Sharma, M.R., Kiel, M.C., Hirokawa, G., Booth, T.M., Spahn, C.M., Grassucci, R.A., Kaji, A., and Frank, J. (2004) Visualization of ribosome-recycling factor on the Escherichia coli 70S ribosome: Functional implications. Proc Natl Acad Sci U S A 101: 8900-8905.

Borgnia, M.J., Shi, D., Zhang, P., and Milne, J.L. (2004) Visualization of alpha-helical features in a density map constructed using 9 molecular images of the 1.8MDa icosahedral core of pyruvate dehydrogenase. J Struct Biol 147: 136-145.

Daberkow, I.H., K.-H.; L., Liu; Rau, W.D.; Tietz, H. (1996) Development and performance of a fast fibre-plate coupled CCD camera at medium energy and image processing system for electron holography. Ultramicroscopy 64: 35-48.

Downing, K.H., and Hendrickson, F.M. (1999) Performance of a 2k CCD camera designed for electron crystallography at $400 \mathrm{kV}$. Ultramicroscopy 75: 215-233.

Faruqi, A.R., and Subramaniam, S. (2000) CCD detectors in high-resolution biological electron microscopy. Q Rev Biophys 33: 1-27.

Frank, J. (1996) Three-dimensional electron microscopy of macromolecular assemblies. San Diego: Academic Press.

Frank, J. (2002) Single-particle imaging of macromolecules by cryo-electron microscopy. Annu Rev Biophys Biomol Struct 31: 303-319.

Glaeser, R.M. (1999) Review: electron crystallography: present excitement, a nod to the past, anticipating the future. J Struct Biol 128: 3-14.

Henderson, R. (1995) The potential and limitations of neutrons, electrons and X-rays for atomic resolution microscopy of unstained biological molecules. Q Rev Biophys 28: 171-193. 
Hesse, J., Hebert, H., and Koeck, P.J. (2000) Evaluation of scanners and CCD cameras for highresolution TEM of protein crystals and single particles. Microsc Res Tech 49: 292-300.

Meyer, R., and Kirkland, A. (2000) Characterisation of the signal and noise transfer of CCD cameras for electron detection. Microsc Res Tech 49: 269 - 280.

Mitsuoka, K., Murata, K., Kimura, Y., Namba, K., and Fujiyoshi, Y. (1997) Examination of the LeafScan 45, a line-illuminating micro-densitometer, for its use in electron crystallography. Ultramicroscopy 68: 109-121.

Sander, B., Golas, M.M., and Stark, H. (2003) Automatic CTF correction for single particles based upon multivariate statistical analysis of individual power spectra. J Struct Biol 142: 392-401.

Sherman, M.B., Brink, J., and Chiu, W. (1996) Performance of a slow-scan CCD camera for macromolecular imaging in a 400 kV electron cryomicroscope. Micron 27: 129-139.

Subramaniam, S., and Milne, J.L. (2004) Three-dimensional electron microscopy at molecular resolution. Annu Rev Biophys Biomol Struct 33: 141-155.

Yonekura, K., Maki-Yonekura, S., and Namba, K. (2003) Complete atomic model of the bacterial flagellar filament by electron cryomicroscopy. Nature 424: 643-650.

Zeitler, E. (1992) The photographic emulsion as analog recorder for electrons. Ultramicroscopy 46: $405-416$.

Zhang, P., Borgnia, M.J., Mooney, P., Shi, D., Pan, M., O'Herron, P., Mao, A., Brogan, D., Milne, J.L., and Subramaniam, S. (2003) Automated image acquisition and processing using a new generation of $4 \mathrm{~K}$ x $4 \mathrm{~K}$ CCD cameras for cryo electron microscopic studies of macromolecular assemblies. J Struct Biol 143: 135-144. 
Figure Legends

Figure 1. (A) Overview (photograph) of the "densitometer robot”, with individual components labeled as follows: 1, stack of films still to be scanned; 2, stack of films that have already been scanned; 3, "hand" with 4 suction-cup fingers that picks up the films; 4, motorized linear track that transports the hand between various locations; 5, "hand" that picks up the top glass plate from the (modified) Nikon film-holder; 6, film holder in the resting position; 7, mechanical unit that pushes the film holder into the Nikon densitometer to start a scan, and pulls it out again to the resting position after a scan has been completed; and 8, the Nikon densitometer. (B) and (C) Close-up views of robot details.

Figure 2. Overview (photograph) of the modified film holder, with key parts labeled as follows: 1, the outer part of the original film holder that is retained, after interior material has been removed; 2, bottom glass plate that replaces the interior material that was removed; 3 , strips of shim stock mounted on either side of the glass plate; 4, barriers placed on either end to block movement of the film when the top glass plate is lifted away; 5, top glass plate (viewed from below); 6, magnets mounted in the corners of the top glass plate; and 7, springs mounted in the top glass plate, which prevent the film from clinging to the top glass plate.

Figure 3. (A) Logarithm of the normalized Nikon output data graphed as a function of the calibrated optical density (OD) values of sub-areas on the JML test film. (The scanned area contained very light and very dark sub-areas, and the scanned data were first normalized to cover the range $10^{-5}$ through 1 . Nevertheless there were still small offsets of the linear functions indicating that the scaling was not fully appropriate, very likely due to slight differences in the representation of the maximum transmittance. These offsets were set to zero.) For all values of 
the parameter Gamma, the logarithm of the output is linear with the OD of each step. The slopes that were found are $1.15,1.03,0.94,0.65,0.48$ for Gamma values $0.9,1.0,1.1,1.6,2.2$, respectively. According to this measurement, the slope is 1.0, and thus the normalized output proportional to the transmittance of film only for the choice of Gamma $=1.03$. (B) Graph showing linearity of the Nikon output versus the amount of electron exposure of photographic film. The value Gamma $=1.0$ was used when digitizing the micrograph. The zero level was obtained from an unexposed sub-area in the number insert area of the micrograph. The small negative offset of the linear fit at zero exposure is likely to be due to a small amount of scattered light in the unexposed sub-area.

Figure 4. Measurement of the modulation transfer function (MTF) of the Nikon Super Coolscan 8000 ED film densitometer. (A) The pure sine-grating patterns of different frequency that are provided on the JML test pattern served as the primary standard for measurement of the MTF. Measurements at specific spatial frequencies in the x-direction (parallel to the linear CCD detector) and in the y-direction (perpendicular to the linear CCD detector) are indicated by triangles and circles, respectively. Note that the MTF decreases more rapidly in the x-direction than it does in the y-direction; possible reasons are discussed in the text. The continuous curves show the apparent, combined MTF of the photographic film and the Nikon densitometer, measured by using a film that was exposed to approximately 0.5 electrons $/ \mu \mathrm{m}^{2}$ (corresponding to about 20 electrons per pixel). Note that the apparent MTF deviates from the true MTF at both low and high frequency; possible reasons are discussed in the text. (B) Smoothed versions of the apparent MTF of film plus the densitometer, in which the low-frequency terms have been truncated (see text for explanation), show a close correspondence to the discrete measurements 
made with the sine-grating test film. The apparent MTF of the Perkin Elmer PDS microdensitometer is also shown for comparison.

Figure 5. Illustration of the major steps involved in partial restoration of the original input, following digitization. (A) The smoothed curves from Figure 4B (apparent MTF curves) are shown along with the smoothed amplitude spectra of the densitometer noise. (B) Twodimensional representation of the apparent MTF. (C) Two-dimensional representation of the spectrum of the densitometer noise. (D) The signal-to-noise $(\mathrm{S} / \mathrm{N})$ ratio of the densitometer, calculated as the ratio of the respective "apparent MTF curves" and "noise curves" that are shown in Panel A. Also shown are the values along the x-direction and the y-direction for a twodimensional Wiener filter function that was constructed to correct for the MTF of the scanner. (E) Two-dimensional representation of the Wiener filter. (F) Apparent MTF curves before and after restoration by applying the Wiener filter.

Figure 6. A practical demonstration of the restoration of the combined output of the film plus densitometer detector-system. The micrograph used in this case was an image of a paraffin crystal supported on a thin carbon film. (A) A 512x512-pixel area of the negative is shown, displayed as optical density. The amplitude spectrum of the displayed area is inset at the upper right. 6 Bragg reflections from the image of the paraffin crystal are faintly visible. (B) The same area of the negative is shown again, but this time after compensation for the MTF of the detector system by means of a Wiener filter. The reflections are clearly visible in the amplitude spectrum. The output spectrum is nearly perfect in all directions out to $2 / 3$ of the Nyquist frequency. As a result all the information about the specimen will be well represented if the highest desired frequency is sampled with three rather than two pixels. 
Figure 7. Technique used to characterize the geometric distortions that are introduced into the digitized image by the Nikon densitometer. (A) Cartoon showing the full area obtained from two scans in which the film was rotated by 90 degrees after the first scan. The first scan is represented by the portrait orientation, A, while the second scan adopts the landscape orientation, B, after being rotated by precisely -90 degrees. Object features within the square region of overlap, C, are in approximately the same orientation. After translational alignment of the region of overlap, a central row and a central column of small boxes were selected, D. These boxes were then used for cross correlation in order to determine the geometric distortions in the Nikon output. (B) Curves showing the displacement of the positions of the 512x512 boxes; see the text for explanation of the notation and a description of how these curves were used to measure the geometric distortions introduced by the densitometer. 